\title{
Vaccination with embryonic stem cells generates effective antitumor immunity against ovarian cancer
}

\author{
ZUJUAN ZHANG, XINHUA CHEN, XIAOHONG CHANG, XUE YE, YI LI and HENG CUI \\ Gynecologic Oncology Center, Peking University People's Hospital, Beijing 100044, P.R. China
}

Received August 8, 2012; Accepted October 12, 2012

DOI: $10.3892 /$ ijmm.2012.1195

\begin{abstract}
To date, only a few studies have suggested that human embryonic stem cells (hESCs) might effectively immunize against colon and lung cancer. The purpose of this study was to investigate the therapeutic potential of hESCs as a vaccine to induce widespread antitumor effects in different animal models and various types of cancer. C57BL/6 mice with ID8 ovarian cancer cell and Fischer 344 rats with NuTu-19 ovarian cancer cell models were used. Fifty-four mice were divided into six groups with nine mice in each group. Each mouse was immunized with pre-inactivated hESCs (H9) or mouse embryonic stem cells (mESCs; IVP-ES1) or ID8 or phosphate-buffered saline (PBS). Twenty-four rats were divided into four groups with six rats in each group, each rat immunized with pre-inactivated hESCs (H9) or NuTu-19 or PBS. After the vaccination, each mouse was challenged with live ID8 cells subcutaneously, and each rat was challenged with live NuTu-19 cells intraperitoneally. We discovered that vaccination of mice with the hESC line H9 and the mESC line IVP-ES1 generated consistent cellular and humoral immune responses against ID8 ovarian cancer. H9 and IVP-ES1 vaccinated mice obtained antitumor immune protection, and H9 vaccinated rats had the longest survival time and least distant metastases. No evidence of side-effects was observed. We also compared the immunogenicity against ovarian cancer between the hESC line, H9, and the mESC line, IVP-ES1, that derived from the inner cell mass in different species. We found that there were no significant differences between them. Furthermore, immunohistochemical staining revealed that several oncogenes and tumor suppressor genes, such as
\end{abstract}

Correspondence to: Professor Yi Li or Professor Heng Cui, Gynecologic Oncology Center, Peking University People's Hospital, 11 South Xizhimen Street, Beijing 100044, P.R. China

E-mail: liyi@pkuph.edu.cn

E-mail: cuiheng20@163.com

Abbreviations: IL-6, interleukin-6; IFN- $\gamma$, interferon- $\gamma$; TNF- $\alpha$, tumor necrosis factor- $\alpha$; PBS, phosphate-buffered saline

Key words: ovarian cancer, embryonic stem cell, antitumor immunity, immunotherapy, cancer vaccine
HER-2, C-myc, p53, and nm23, were expressed in H9, many of which were also shared by ovarian cancer. hESC vaccines can induce antitumor effects in two animal models and in ovarian cancer, indicating that the activity of the vaccine is universal, and, more importantly, it is safe.

\section{Introduction}

Tumor vaccines are currently very common in terms of development of active immunotherapy. A major question is whether or not a vaccine that can break immune tolerance and is widely applicable to many patients really exists. A few recent studies have shown that human embryonic stem cells (hESCs) have the ability to become the new vaccine of choice. Li et al (1) demonstrated the capacity of hESCs to effectively immunize against murine colon cancer for the first time. This was further supported by an additional study (2).

However, results on colon and lung cancer only have been reported, and only in mouse models. Furthermore, the embryonic stem cells (ESCs) and the animals were the same species in the lung cancer model. Whether antitumor effects of ESCs are occasional or universal, whether their antitumor effects are present in some specific types of cancer or in a broad spectrum of different types of cancer, and whether hESCs can overcome species obstacles and induce the same antitumor effects in different animal species and different original cancer tissue, remains unknown. In this study, we chose hESCs and mouse ESCs (mESCs), and administered them as vaccines in ovarian cancer in a mouse and rat model. We hypothesized that undifferentiated human stem cells could be a proper vaccine for ovarian cancer and immunize mice and rats to generate an immune response. hESCs were indeed able to induce a broad spectrum of both immune responses and clinical responses against ovarian carcinoma without evidence of inducing autoimmune diseases or side-effects.

\section{Materials and methods}

Cell lines. The hESC line H9 was provided by Professor MingXiao Ding (School of Life Sciences, Peking University). These cells were grown in hESC medium. The mESC line IVP-ES1 was a generous gift from Qi Zhou (Institute of Zoology of Chinese Academy of Sciences). Cells were cultured in mESC medium (Beijing Huasen Biotechnology). The ID8 cell line (a mouse ovarian epithelial papillary serous adenocarcinoma 
A

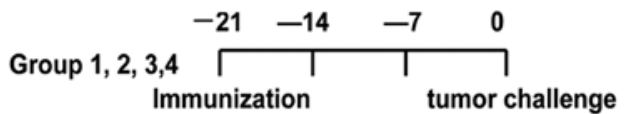

B

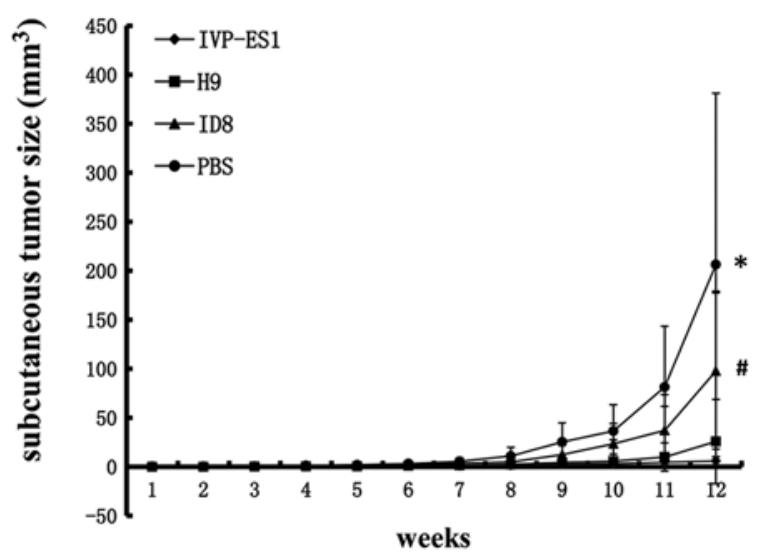

C

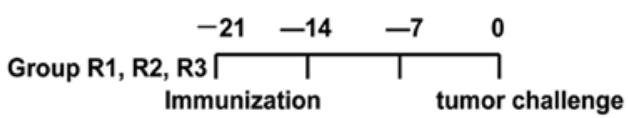

D

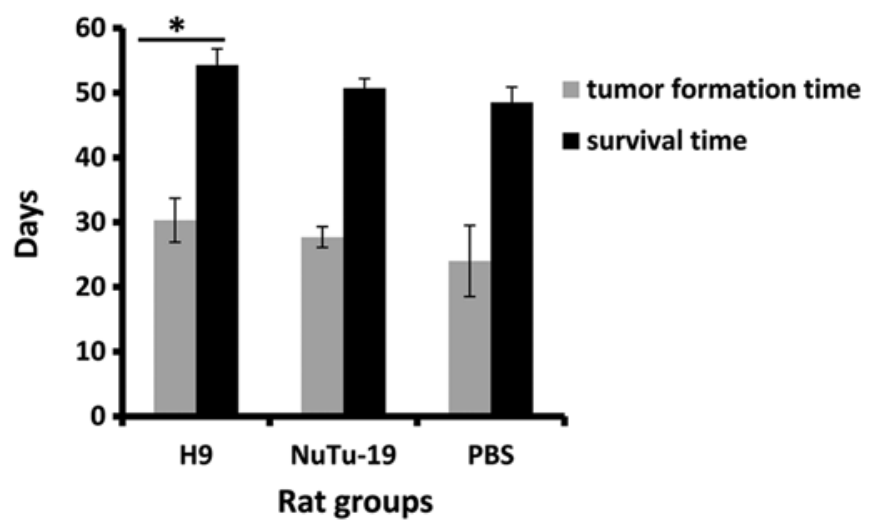

Figure 1. Undifferentiated ESCs exhibit potent antitumor activity in mouse and rat ovarian cancer models. (A) Mice in Groups 1, 2, 3 and 4 received subcutaneous vaccination $5 \times 10^{6}$ inactivated H9, IVP-ES1, ID8 and PBS, respectively, three times at a one-week interval, and then alive ID8 cells $\left(5 \times 10^{6}\right)$ were challenged one week after the final vaccination. (B) Tumor formation time and growth rates in mESCs (IVP-ES1, Group 1) and hESCs (H9, Group 2) were longer than that in Group 3 (ID8) and in Group 4 (PBS). The sizes of the subcutaneous tumors were 5.9 $\pm 4.2,25.7 \pm 43.0,97.6 \pm 79.7,206.3 \pm 174.8 \mathrm{~mm}^{3}$ $\left({ }^{*} \mathrm{P}<0.05\right)$, respectively, at the end of week 12 . Furthermore, there was no statistically significant difference in the antitumor effects between IVP-ES1 and H9 cells. (C) Rats in Groups R1, R2 and R3 received 1x107 inactivated H9, NuTu-19 and PBS vaccination subcutaneously three times at one-week intervals, and one week after the final vaccination; $1 \times 10^{6}$ alive NuTu-19 cells were intraperitoneally challenged. (D) hESC vaccination prolonged survival in the rat model. Tumor formation times in H9, NuTu-19 and PBS were $30.3 \pm 3.4,27.7 \pm 1.6$ and $22.0 \pm 5.5$ days $\left({ }^{*} \mathrm{P}<0.05\right)$ respectively; the survival times were $54.3 \pm 2.5,50.7 \pm 1.5$ and $48.5 \pm 2.4$ days $\left({ }^{*} \mathrm{P}<0.05\right)$, respectively. $\mathrm{H} 9$ vaccination resulted in a markedly longer survival time compared with the control groups.

cell line) was a generous gift from K.F. Roby (Center for Reproductive Sciences, University of Kansas Medical Center, Kansas City, KS, USA). ID8 cells were cultured in DMEM supplemented with 5\% fetal bovine serum. NuTu-19 cells, a Fischer 344 rat-derived epithelial ovarian carcinoma cell line, was grown in RPMI-1640 medium containing 10\% newborn calf serum (NBCS). H9 and IVP-ES1 cells were irradiated with 15 Gy gamma-ray before vaccination. ID8 and NuTu-19 cells were incubated with $10 \mu \mathrm{g} / \mathrm{ml}$ mitomycin $\mathrm{C}$ for $3 \mathrm{~h}$ at $37^{\circ} \mathrm{C}$. Additionally, one plate of $\mathrm{H} 9$ cells was processed for paraffin embedding, $3 \mu \mathrm{m}$ sections were prepared, and immunohistochemistry was performed for several tumor antigens and genes and examined with a microscope.

Animals. Specific pathogen-free C57BL/6 female mice (20-25 g) and Fischer 344 female rats (100-125 g) were obtained from the Academy of Military Medical Sciences (Beijing, China) and housed in a pathogen-free animal facility at Peking University People's Hospital, Beijing, China. Treatment and care of the animals were in accordance with Institutional Guidelines and the Animal Welfare Assurance Act. The experimental protocol for the use of the animals for these studies was approved by the Institutional Laboratory Animal Care and Use Committee at Peking University People's Hospital (81072141).

Mouse immunization protocol. C57BL/6 mice were randomly divided into six groups, each group containing nine mice. Groups 1, 2, 3 and 4 received subcutaneous vaccination in the right flank with pre-inactivated IVP-ES1 $\left(5 \times 10^{6}\right)$ or H9 $\left(5 \times 10^{6}\right)$ or ID8 $\left(5 \times 10^{6}\right)$ or phosphate-buffered saline (PBS), respectively, repeated three times at a one-week interval, and alive ID8 cells $\left(5 \times 10^{6}\right)$ were inoculated subcutaneously in the left flank one week after the final vaccination. Groups 5 and 6 received subcutaneous vaccination with pre-irradiated $\mathrm{H} 9$ $\left(5 \times 10^{6}\right)$ or IVP-ES1 $\left(5 \times 10^{6}\right)$ three times at a one-week interval, two weeks after the final vaccination. Orbital venous blood was collected to obtain the serum as the primary antibodies for western blotting (Fig. 1A).

Tumor growth was monitored every week using digital calipers to measure both the longitudinal ( $\mathrm{L}, \mathrm{mm})$ and transverse $(\mathrm{W}, \mathrm{mm})$ diameters. Tumor volume $\left(\mathrm{LxW}^{2} / 2, \mathrm{~mm}^{3}\right)$ was plotted. Mice were also monitored for the following general health indicators: overall behavior, feeding, neuromuscular tone, body weight and appearance of fur, particularly after immunization. The endpoint for this study was one diameter of tumor $\geq 10 \mathrm{~mm}$, at which point mice were euthanized. The primary tumor was also excised and weighed after the mice were sacrificed.

Rat immunization protocol. Fischer 344 rats were randomly divided into four groups with each group containing six rats. Group R1, R2, R3 received subcutaneous vaccination with preinactivated H9 $\left(1 \times 10^{7}\right)$ or NuTu-19 $\left(1 \times 10^{7}\right)$ or PBS, respectively, repeated three times at one-week intervals, and alive $\mathrm{NuTu}-19$ cells $\left(1 \times 10^{6}\right)$ were intraperitoneally inoculated one week after the final vaccination; Group R4 received subcutaneous vaccination with pre-irradiated $\mathrm{H} 9\left(1 \times 10^{7}\right)$ only, repeated six times at one-week intervals. Side-effects were observed in this group, including dynamic complete blood count (CBC) tests, liver and kidney function, including glutamic-oxalacetic transaminase (GOT); glutamic-pyruvic transaminase (GPT); 
Table I. Human ESC vaccination inhibits tumor metastasis in a rat model.

\begin{tabular}{|c|c|c|c|}
\hline & Group R1 (H9) & Group R2 (NuTu-19) & Group R3 (PBS) \\
\hline Metastatic organs & $\begin{array}{l}\text { Diaphragm, peritoneal wall, } \\
\text { intestine, mesentery, } \\
\text { omentum }\end{array}$ & $\begin{array}{c}\text { Diaphragm, peritoneal wall, } \\
\text { intestine, mesentery, } \\
\text { omentum }\end{array}$ & $\begin{array}{l}\text { Diaphragm, peritoneal wall, } \\
\text { intestine, mesentery, } \\
\text { omentum, kidney, } \\
\text { liver surface and parenchyma lung }\end{array}$ \\
\hline $\begin{array}{l}\text { Liver and parenchyma } \\
\text { metastasis }\end{array}$ & $0 \%(0 / 6)$ & $16.7 \%(1 / 6)$ & $33.3 \%(2 / 6)$ \\
\hline Lung metastasis & $0 \%(0 / 6)$ & $33.3 \%(2 / 6)$ & $50.0 \%(3 / 6)$ \\
\hline $\begin{array}{l}\text { Metastatic tumor } \\
\text { size }>0.5 \mathrm{~mm}^{3}\end{array}$ & $16.7 \%(1 / 6)$ & $66.7 \%(4 / 6)$ & $83.3 \%(5 / 6)$ \\
\hline
\end{tabular}

ESC, embryonic stem cell; PBS, phosphate-buffered saline.

creatinine (CRE) (Peking University Health Science Center, PUHSC). Figs. $1 \mathrm{C}$ and $3 \mathrm{~A}$ show the scheme of ESC immunization and tumor inoculation.

All animal health conditions were monitored daily. Tumor formation and survival times were observed in Groups R1, $\mathrm{R} 2$ and R3. Tumor metastasis was recorded by counting the numbers and sizes of tumor foci on each organ.

Th1/Th2/Th17 cytokines. Fresh blood from all mouse groups was obtained before sacrificing by anesthesia. The serum levels of interleukin-2 (IL-2), IL-4, IL-6, IL-10, IL-17, IFN- $\gamma$, tumor necrosis factor- $\alpha(\mathrm{TNF}-\alpha)$ were tested by cytometric bead array (CBA) kits (Becton-Dickinson) in accordance with the instructions and analyzed by BD Cytometric Bead Array Analysis.

Western blotting. H9, IVP-ES1 and ID8 cells were rinsed with PBS and lysed in $100 \mu 1$ Laemmli sample buffer. The samples were separated by electrophoresis on a $10 \%$ denaturing and reducing SDS-polyacrylamide gel. They were then transferred onto an Immobilon-polyvinylidene fluoride membrane (Millipore). Membranes were blocked in 5\% skim milk for $1 \mathrm{~h}$ and then incubated with the indicated primary antibodies at 1:50 dilution of sera from either naïve or different cell-immunized mice overnight. This was followed by incubation with the appropriate secondary antibodies, goat anti-mice $(1: 3,000$ dilution) for $1 \mathrm{~h} 30 \mathrm{~min}$. Specific proteins were detected using enhanced chemiluminescence.

Side-effects on the blood system, liver and kidney function. Rat orbital venous blood collection was performed after the first vaccination. The following punctures were performed at the interval of vaccination twice at a two week interval in Groups R1, R2, R3 and three times at a two week interval in Group R4. Both whole blood and serum underwent CBC analysis, including white blood count (WBC), red blood cell (RBC), hemoglobin (HGB), and platelets (PLT), and assay of biochemical function indicator of liver and kidney, including GOT, GPT, CRE (Peking University Health Science Center, PUHSC).
Statistical analysis. Time to death (or euthanization) was completed using Kaplan-Meier estimates of survival time. Equality of survival was determined using the two-sided logrank test. Median survival times with $95 \%$ confidence limits for each treatment group were also determined. Statistical significance of differences in tumor growth rates was determined by ANOVA test analysis of variance using SPSS 20.0. Most data are presented as the means \pm SD, and a P-value $<0.05$ was considered to indicate statistically significant differences.

\section{Results}

Inhibition of tumor formation in the mouse model. We used a well-established C57BL/6 mice epithelial ovarian cancer model (3). After challenging with alive ID8 cells, the kinetics of tumor growth were closely monitored. Tumor formation times and growth rates in mESCs (IVP-ES1, Group 1) and hESCs (H9, Group 2) were longer than that in Group 3 (ID8, positive control) and in Group 4 (PBS, negative control). At the end point, the sizes of the subcutaneous tumors were $5.9 \pm 4.2,25.7 \pm 43.0$, 97.6 $\pm 79.7,206.3 \pm 174.8 \mathrm{~mm}^{3}(\mathrm{P}<0.05)$, respectively, (Fig. 1B). We concluded that immunization with undifferentiated human or mouse ESCs can generate effective antitumor activity.

Prolonged survival and inhibition of tumor metastasis in the rat model. In the Fischer 344 rat epithelial ovarian cancer model (4), the tumor formation time in hESCs (H9, Group R1) was longer than that in the two control groups. Tumor formation times in Groups R1, R2, R3 were 30.3 \pm 3.4 , 27.7 \pm 1.6 and $22.0 \pm 5.5$ days $(\mathrm{P}<0.05)$ respectively; the survival times were $54.3 \pm 2.5,50.7 \pm 1.5$ and $48.5 \pm 2.4$ days $(\mathrm{P}<0.05)$ respectively. $\mathrm{H} 9$ vaccination resulted in a markedly longer survival time compared with the control groups $(\mathrm{P}<0.05)$ (Fig. 1D). Furthermore, vaccination with hESCs resulted in a retardation of tumor growth in average tumor size compared to the control group (Table I). Upon gross visual inspection of the peritoneum, numerous tumors were observed in control rats. However, there were fewer tumors in the $\mathrm{H} 9$ vaccinated rats (Table I). The results suggested that the H9 vaccination group induced obvious antitumor immunity and rejected tumor 
A

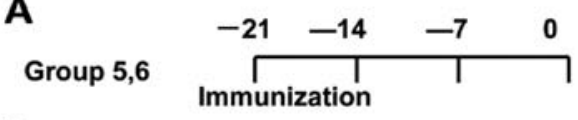

B
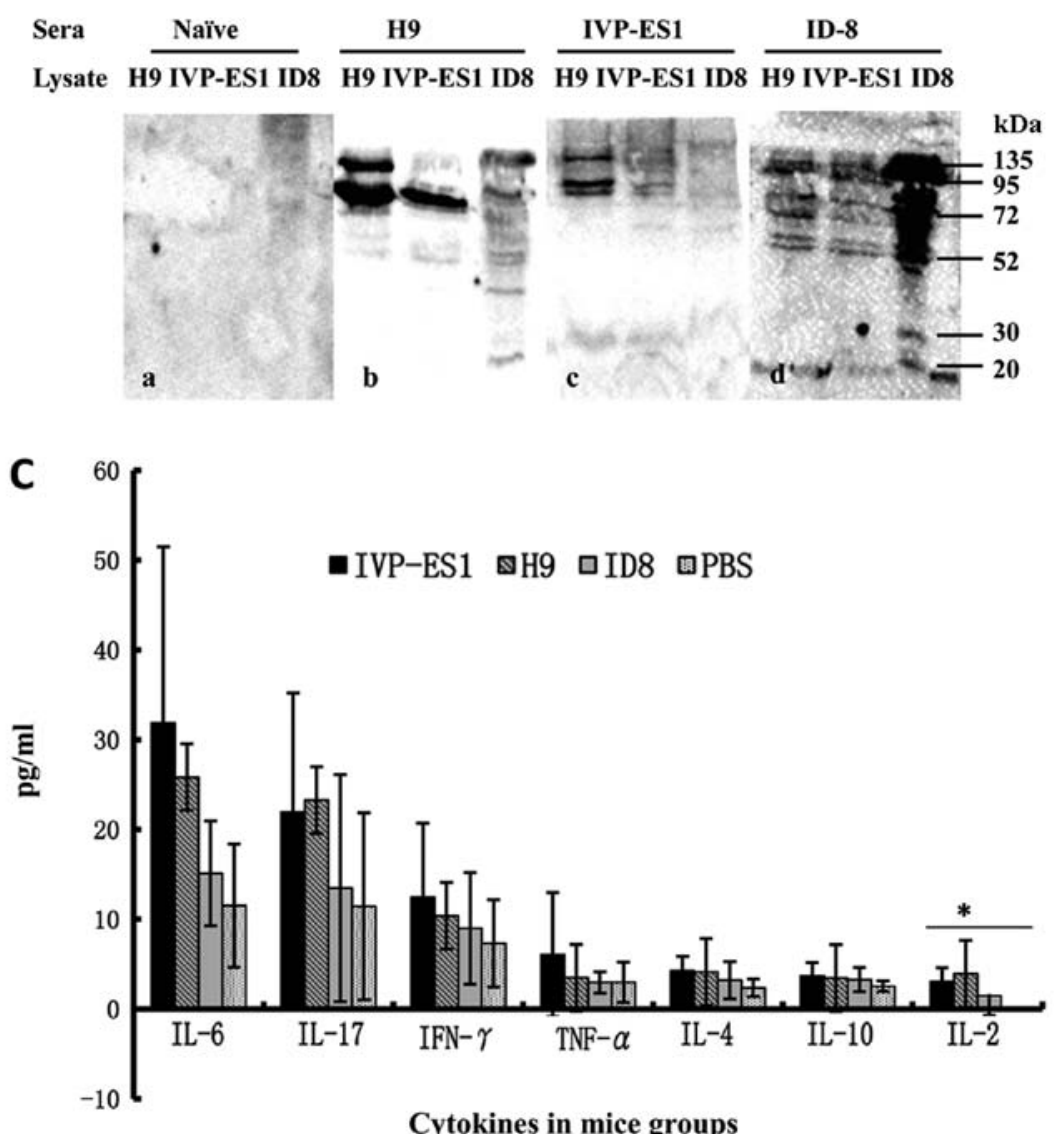

Figure 2. Vaccination with hESCs and mESCs induced both T-cell and antibody response against ovarian cancer. (A) Groups 5 and 6 received subcutaneous vaccination of inactivated $\mathrm{H} 9$ and IVP-ES1, respectively, three times at a one-week interval, two weeks after the final vaccination; orbital venous blood was collected. (B) Immunization with H9 and IVP-ES1 generated cross-reactive antibody against ID8 ovarian cancer. Western blot analysis was performed with sera from immunized mice against total cell lysate of ID8 and undifferentiated H9, IVP-ES1 cells. Numbers indicate the molecular weight marker (kDa), (C) Mice were sacrificed after the final immunization for the enumeration of the frequency of IL-6, IL-17, IFN- $\gamma$, TNF- $\alpha$, IL-4, IL-10 and IL-2. Irradiated ID8 tumor cells served as target cells, along with PBS as a negative control and ESCs as a positive control. There were nine mice/group. IL- 2 , ${ }^{*} \mathrm{P}<0.05$.

masses from proliferation and development compared to the control groups. In the R1 group, metastatic lesions were found in the diaphragm, peritoneal wall, intestine, mesentery and omentum. Moreover, the metastatic tumor size $\geq 0.5 \mathrm{~mm}^{3}$ was only found in one rat; however, in Groups R2 and R3 the metastasis included those in R1, and transferred to the kidney, liver surface parenchyma and lung, the metastatic tumor size $\geq 0.5 \mathrm{~mm}^{3}$ was found in five rats in these two groups. Furthermore, lung and liver parenchyma metastases were found in eight rats in Groups R2 and R3, however, no case developed such distant metastasis as in Group R1.

Comparison of the immunogenicity between undifferentiated hESCs and mESCs in the murine model. We compared hESCs and mESCs in the same ovarian cancer mouse model. The primary antitumor responses of H9 and IVP-ES1 were evaluated according to the same immunization procedure. We demonstrated that immunization with undifferentiated H9 and IVP-ES1 cells could inhibit tumor growth compared to two control groups. Furthermore, there was no statistically significant difference in the antitumor effects between IVP-ES1 and H9 cells (Fig. 1B). These results strongly suggest that the immunogenicity of hESCs is similar to mESCs to a large extent, and hESCs can induce the same antitumor effects as mESCs.

Vaccination with ESCs induces antibody response against murine ovarian cancer. Sera from non-immunized naïve mice did not react with H9, IVP-ES1 or ID8. However, we demonstrated that sera from ID8 immunized rats were able to recognize multiple proteins in ID8, H9 as well as IVP-ES1 lysates by western blot analysis (Fig. 2A and B). Moreover, prominent 135, 95, 72, 52, $30 \mathrm{kDa}$ molecules were recognized by sera from H9-immunized, IVP-ES1-immunized mice and detected in ID8 ovarian cancer cells (Fig. 2A and B). This suggested that an antitumor antibody response was produced after H9, IVP-ES1 immunization and that there existed shared antigens among ID8 and undifferentiated H9, IVP-ES1 cells. 
A

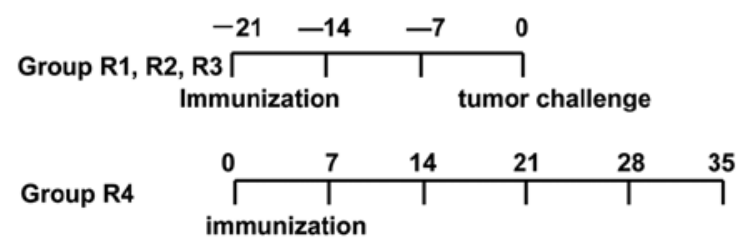

B

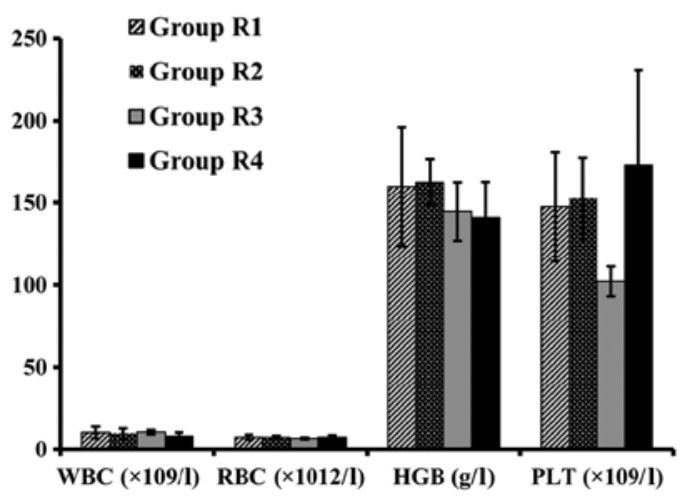

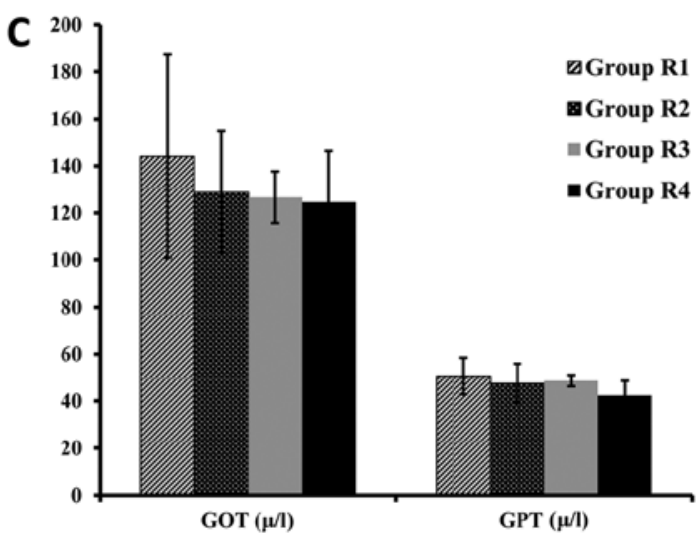

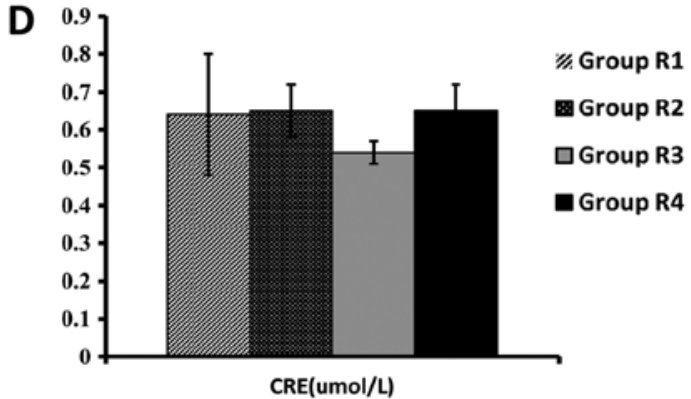

Figure 3. Immunization with hESCs did not result in significant hematologic toxicity or side-effects. (A) Rats in Group R4 received subcutaneous vaccination with pre-irradiated $\mathrm{H} 9\left(1 \times 10^{7}\right)$ only, six times at one-week intervals, two weeks after the final vaccination; orbital venous blood was collected. (B) CBC tests, including WBC, RBC, HGB and PLT showed no significant differences in each group of rats. (C and D) Blood serum enzymes in liver and kidney, GOT, GPT and CRE showed no significant differences in each group of rats.

Vaccination with ESCs induces cell-based immunity. We next examined whether hESC and mESC immunization induced cell-based antitumor immune response against ID8. We used the Th1/Th2/Th17 CBA analysis kit (Becton-Dickinson) to test and compare cytokine levels in each group, including IL-2, IL-4, IL-6, IL-10, IL-17, TNF- $\alpha$ and IFN- $\gamma$. We found there was an increasing trend of Th1/Th2/Th17 cytokine levels in the hESC and mESC vaccinated groups compared with the control groups. We did not find any difference of IL-4, IL-6, IL-10, IL-17, TNF- $\alpha$, IFN- $\gamma(\mathrm{P}>0.05)$ levels among the four groups except for IL-2. The IL-2 levels in each group were $3.9 \pm 1.7,3.1 \pm 1.5,1.5 \pm 2.1,0(\mathrm{pg} / \mathrm{ml})$, separately $(\mathrm{P}<0.05)$ (Fig. 2A and C). It may be the case that cell-mediated immunity is so complicated that other mechanisms and factors may be involved (5).

Immunization with hESCs does not result in significant hematologic toxicity or side-effects. An important consideration for stem cell-based vaccines is the possibility of breaking immune tolerance against self-antigens, such as cross-reactive antibodies against the hematologic system and side-effects in the liver and kidney. This question also has bearing on the application of stem cells for regenerative medicine in an immunocompetent host. As an index for inhibition in the hematologic system and side-effects in important organs, we performed dynamic $\mathrm{CBC}$ tests, and the levels of several blood serum enzymes in the sera of rats that were immunized with PBS, H9 or NuTu-19 cells were determined, as side-effects are easier to be observed and more blood is available in rats than in mice. We used the vaccine Group R4 as the positive control, since this group underwent inoculation with $\mathrm{H} 9$ cells only (Fig. 3A). CBC tests were in the normal range and showed no difference among rats immunized with PBS, H9 or NuTu-19 cells (Fig. 3B). Creatinine and serum enzyme levels were normal in control and $\mathrm{H} 9$ cell-immunized rats (Fig. 3C and D). However, four cases showed extraordinarily elevated levels $>100$ fold compared to controls. Three rats treated with PBS and all rats treated with pre-inactivated mitotic NuTu-19 cells were in a late state and showed signs of severe cachexia and liver and kidney toxicities, including metastases to both organs. More importantly, we did not observe any abnormalities in the weight, hair, joint swelling and neuromuscular tension of the animals.

hESCs express some non-specific ovarian tumor antigens. Using immunohistochemical methods to screen tumor markers, we found that several oncogenes, tumor suppressor genes, and metastasis-related genes had high expression in hESCs (H9). Such markers included HER-2 (+), C-myc (++), p53 (++), nm23 (+++). We also identified some tumor markers, such as NSE (+), PTEN $(+), \operatorname{PLAP}( \pm), \operatorname{HPL}( \pm), \mathrm{CK}(++), \mathrm{MBP}$ $(++)$, and Vimentin $(+++)$ (Fig. 4). hESCs express a broad spectrum of tumor antigens, markers, and genes related to tumor growth and metastasis.

Results were interpreted by two pathologists independently, and a mean percentage of positive cells was determined in at least five areas at $\mathrm{x} 400$ and assigned to one of four categories: $(-),<5 \%$; (+), 5-25\%; (++) 25-50\%; and (+++), $>50 \%$. 

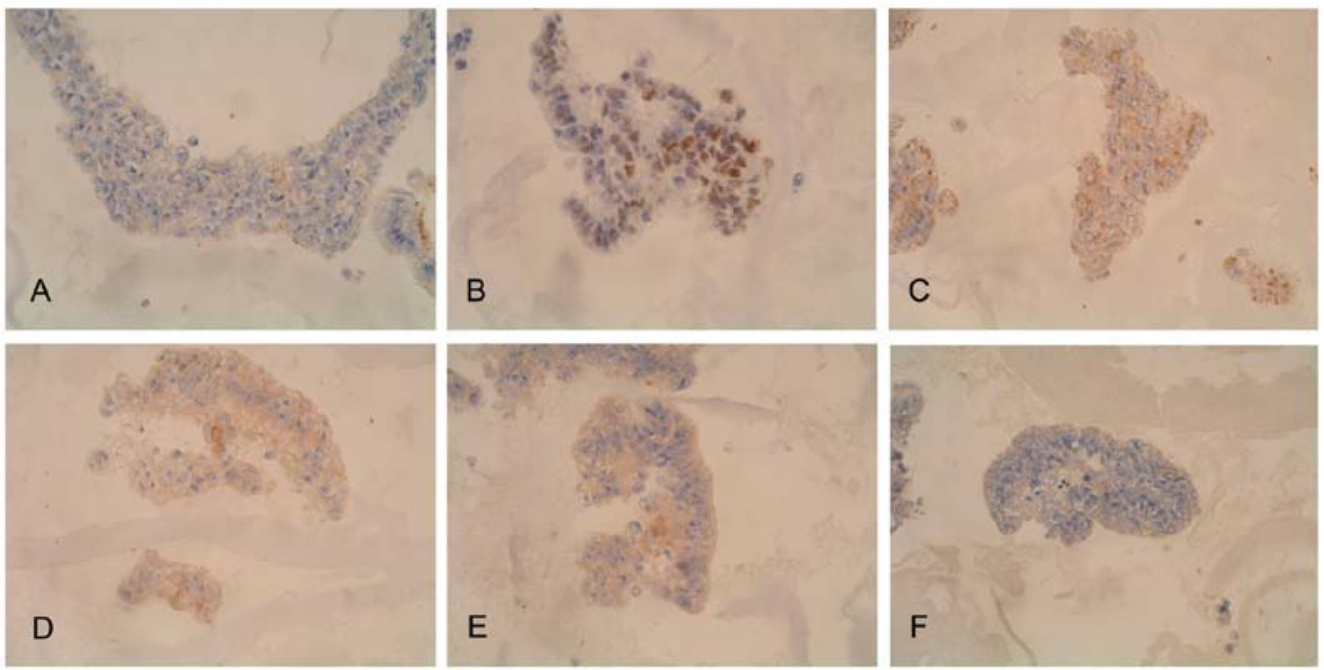

Figure 4. Immunohistochemical staining of tissue showing representative expression of each protein. (A) HER-2 (+), cell membrane staining (x200); (B) P53 (++), nuclear staining (x200); (C) C-myc (++), cytoplasmic staining (x200); (D) nm23 (+++), cell membrane and cytoplasmic staining (x200); (E) PTEN (+), cytoplasmic staining (x200); (F) PLAP (+/-), cell membrane staining (x200).

\section{Discussion}

Embryonic stem cells have obvious immune antitumor effects in mouse colon and lung cancer, a prospect that is encouraging. Li et al (1) demonstrated the capacity of human embryonic stem cells (hESCs) to effectively immunize against murine colon cancer for the first time. This was further supported by an additional study in which a combination of carbon nanotubes and embryonic stem cells (ESCs) successfully provided activation of antitumor immunity, leading to an impressive suppression of proliferation and development of malignant colon tumors (2). Furthermore, Dong et al (6) demonstrated that administration of ESCs could prevent and control the proliferation and development of lung cancer. However, these studies only examined colon and lung cancer, while Li et al (1) reported only devised human iPS cells (induced pluripotent stem cells) as a control group without mouse ESCs (mESCs). Dong et al (6) reported administering mESCs in mouse lung cancer without hESCs as a control group, therefore it remains uncertain whether hESCs can overcome species obstacles and induce the same antitumor effects in different animal species and different original cancer tissue.

In this study, we used both a mouse and a rat ovarian cancer animal model, particularly the rat model, since rats have more similarities to human disease and make it is easier to observe side-effects. Furthermore, we devised hESCs and mESCs in the same mouse model, and compared the antitumor effects of human and mouse ESCs. In this study, we proved that undifferentiated hESCs could be a novel and safe vaccine for ovarian cancer both in the mouse and the rat model. Additionally, this study also demonstrated the potential concerns associated with cross-species obstacles.

In our study, we found mESCs (IVP-ES1) were able to immunize naïve mice against challenge with live murine ovarian carcinoma cells, while hESCs (H9) were able to immunize naïve mice and rats against challenge with a lethal dose of live ovarian carcinoma cells. We also discovered administration of both hESCs and mESCs could generate effective antitumor effects and protect animals from tumor proliferation and/or further development. Moreover, in the mouse ovarian cancer model, antitumor effects of human and mice ESCs were quite similar. We also found that the survival times in the vaccine group in rats were much longer than those in the control groups. We speculated that hESCs could suppress tumor proliferation and prolong the survival time.

More importantly, rats are a relatively bigger animal model compared to mice, making it easier to find the side-effects of therapy compared with mice; the dynamic blood sampling is also more feasible in rats, so we performed dynamic CBC tests, and the levels of several blood serum enzymes in the sera of rats in all groups. We did not find marrow suppression or any damage to liver and kidney function. Additionally, we did not observe any abnormalities in the weight, hair, joint swelling and neuromuscular tension of the animals. We did not observe any significant side-effects in the hESC-immunized rats and mice. Immunized rats and mice were generally healthy without clinical evidence of autoimmune diseases.

We examined the potential mechanism of tumor rejection by stem cell-immunized mice. We observed both humoral and cell-based immunity. However, it is difficult to attribute responsibility for tumor rejection to a single mechanism. It is thus not surprising that not all H9 and IVP-ES1 cells induced significant numbers of IL-4, IL-6, IL-10, IL-17A, TNF- $\alpha$ and IFN- $\gamma$, however, there is an increasing trend in ES vaccinated mouse serum. Furthermore, IL-2 levels in serum in H9 and IVP-ES1 vaccinated mice was higher than in the control groups, which showed statistically significant differences. IL-2 and IL- 4 produced splenocytes and TNF- $\alpha$ against ID8. hESCs induced growth and metastasis suppression in rat ovarian cancer cell lines. However, such inhibition does not appear to be mediated only by mouse cytokines IL-2, IL-4, IL-6, IL-10, IL-17A, TNF- $\alpha$ and IFN- $\gamma$. There may still be other dynamic immunological factors implicated in the administration of the hESC vaccine processes. The unique properties of hESCs may provide a novel approach for therapeutic options for the management of cancer. 
Previous studies support the hypothesis that tumor-embryonic antigens (oncofetal antigens) are expressed in cancer cells and in embryonic material (1-3). Thus, anti-embryonic antigens play a role in the antitumor immune response through crossimmune reactivity (7). The exact antigens shared by hESCs, mESCs and ID8 ovarian carcinoma cells remain to be identified. It is possible that the antigens that were reactive with the H9, IVP-ES1 and ID8 immune sera were oncofetal antigens present in these cells. Stem cell immunization might trigger an immune response against these gene products that are also expressed by tumor cells. Additionally, the immune response against H9, IVP-ES1 could lead to antigenic spread to induce protective immunity against ID8 unique tumor antigens, a concept akin to that proposed to explain the efficiency of xenogeneic antigen immunization (8). We found cross-reactive proteins among H9, IVP-ES1 and ID8 by western blotting. The ability to separate and purify these proteins in order to find the exact antigen and to explore the antitumor mechanism of ESCs are both questions worthy of further exploration.

We also screened tumor-embryonic antigens and several genes related to tumors by immunohistochemical methods. We found several genes or markers related to tumorigenesis, tumor growth and metastasis. Many of these were involved in critical tumor signal transduction pathways. For example, nm23 and HER-2 were negatively correlated with tumor metastasis and prognosis. Notably, HER-2 has been exploited as a promising candidate for peptide-based cancer vaccines (9-12). PTEN, p53, and c-myc, which are all well known to play important roles in carcinogenesis, have also been shown to be associated with prognosis (13-18). These results show that hESCs do express a broad spectrum of tumor markers, many of which are also shared by ovarian cancer. This provides us with a basis for examining tumor markers for tumor immunotherapy.

However, additional follow-up studies are needed before hESC-based cancer vaccines move into clinical testing. In a broad context, our study has raised a number of intriguing questions that deserve further research. For example, with further optimization, could an hESC-based vaccination strategy be effective against pre-established cancer? ESCs have been proven to be effective vaccines in colon, lung, and, here, in ovarian cancer. Animal models used for these studies include mice and rats, suggesting that cross-species obstacles may not be particularly problematic and that hESCs may produce a broad spectrum of antitumor effects. Despite our progress, there is still much that remains unknown, such as the exact mechanism of the antitumor effects of ESCs. Although we detected some cross-reactivity of antigens between ESCs and tumor cells, a major question is how to identify and purify these cross antigens, what they are exactly, if they are involved in tumor metastasis, and whether cancer stem cells can be used as vaccines since they might share more cross-antigens with the cancer cells of origin.

In summary, we demonstrated the capacity of hESCs to effectively immunize against mouse and rat ovarian cancer, and no side-effects were observed. hESC vaccines can induce similar antitumor effects in different species and in different original cancer tissue, which indicates that the activity of the vaccine is universal. The unique properties of ES cells may provide a novel approach for therapeutic options for the management of ovarian cancer.

\section{Acknowledgements}

This study was supported by the National Natural Science Foundation of China (grant no. 81072141, to Y.L.), the Research and Development foundation in Peking University People's Hospital (grant no. RDB2010-09, to Y.L.). The authors thank Professor Katherine F. Roby for providing the ID8 cells, University of Kansas Medical Center. The authors also thank Professor Ming-Xiao Ding, Dr Dong-Hui Zhang and Dr Hai-Song Liu, School of Life Sciences, Peking University, for their kind assistance in the processing of human embryonic stem cell culture.

\section{References}

1. Li Y, Zeng H, Xu RH, Liu B and Li Z: Vaccination with human pluripotent stem cells generates a broad spectrum of immunological and clinical responses against colon cancer. Stem Cells 27: 3103-3111, 2009

2. Mocan T and Iancu C: Effective colon cancer prophylaxis in mice using embryonic stem cells and carbon nanotubes. Int $\mathrm{J}$ Nanomed 6: 1945-1954, 2011.

3. Roby KF, Taylor CC, Sweetwood JP, et al: Development of a syngeneic mouse model for events related to ovarian cancer. Carcinogenesis 21: 585-591, 2000.

4. Rose GS, Tocco LM, Granger GA, et al: Development and characterization of a clinically useful animal model of epithelial ovarian cancer in the Fischer 344 rat. Am J Obstet Gynecol 175: 593-599, 1996.

5. Nolz JC, Starbeck-Miller GR and Harty JT: Naive, effector and memory CD8 T-cell trafficking: parallels and distinctions. Immunotherapy 3: 1223-1233, 2011.

6. Dong W, Du J, Shen H, et al: Administration of embryonic stem cells generates effective antitumor immunity in mice with minor and heavy tumor load. Cancer Immunol Immunother 59: 1697-1705, 2010.

7. Brewer BG, Mitchell RA, Harandi A and Eaton JW: Embryonic vaccines against cancer: an early history. Exp Mol Pathol 86: 192-197, 2009.

8. Huebener N, Fest S, Hilt K, et al: Xenogeneic immunization with human tyrosine hydroxylase DNA vaccines suppresses growth of established neuroblastoma. Mol Cancer Ther 8: 2392-2401, 2009.

9. Niitsu N, Nakamine H and Okamoto M: Expression of nm23-H1 is associated with poor prognosis in peripheral T-cell lymphoma, not otherwise specified. Clin Cancer Res 17: 2893-2899, 2011.

10. Wang PH, Yi YC, Tsai HT, et al: Significant association of genetic polymorphism of human nonmetastatic clone 23 type 1 gene with an increased risk of endometrial cancer. Gynecol Oncol 119: 70-75, 2010.

11. Kedrin D, Wyckoff J, Boimel PJ, et al: ERBB1 and ERBB2 have distinct functions in tumor cell invasion and intravasation. Clin Cancer Res 15: 3733-3739, 2009.

12. Lekka E, Gritzapis AD, Perez SA, et al: Identification and characterization of a HER-2/neu epitope as a potential target for cancer immunotherapy. Cancer Immunol Immunother 59: 715-727, 2010.

13. Schade B, Rao T, Dourdin N, et al: PTEN deficiency in a luminal ErbB-2 mouse model results in dramatic acceleration of mammary tumorigenesis and metastasis. J Biol Chem 284: 19018-19026, 2009.

14. Rodriguez OC, Lai EW, Vissapragada S, et al: A reduction in Pten tumor suppressor activity promotes ErbB-2-induced mouse prostate adenocarcinoma formation through the activation of signaling cascades downstream of PDK1. Am J Pathol 174: 2051-2060, 2009.

15. Huang S, Benavente S, Armstrong EA, Li C, Wheeler DL and Harari PM: p53 modulates acquired resistance to EGFR inhibitors and radiation. Cancer Res 71: 7071-7079, 2011.

16. Sasaki Y, Negishi $\mathrm{H}$, Idogawa $\mathrm{M}$, et al: $\mathrm{p} 53$ negatively regulates the hepatoma growth factor HDGF. Cancer Res 71: 7038-7047, 2011.

17. Yasojima H, Shimomura A, Naoi Y, et al: Association between c-myc amplification and pathological complete response to neoadjuvant chemotherapy in breast cancer. Eur J Cancer 47: 1779-1788, 2011.

18. Chen Y, Xu J, Borowicz S, Collins C, Huo D and Olopade OI: c-Myc activates BRCA1 gene expression through distal promoter elements in breast cancer cells. BMC Cancer 11: 246, 2011. 